Journal of

Molecular Microbiology

and Biotechnology

\title{
Global Transcriptional Analysis of Bacillus licheniformis Reveals an Overlap between Heat Shock and Iron Limitation Stimulon
}

\author{
Allan K. Nielsen ${ }^{a} \quad$ Anne Breüner ${ }^{a} \quad$ Marcin Krzystanek $^{a} \quad$ Jens T. Andersen ${ }^{a}$ \\ Thomas A. Poulsen ${ }^{a}$ Peter B. Olsen ${ }^{a} \quad$ Ivan Mijakovic $^{b} \quad$ Michael D. Rasmussen $^{a}$ \\ ${ }^{a}$ Molecular Biotechnology, Novozymes A/S, Bagsværd, Denmark; ${ }^{b}$ Micalis UMR 1319, AgroParisTech/INRA, \\ Jouy-en-Josas, France
}

\section{Key Words}

Heat shock • Iron uptake - Transcriptomics - Fur regulator • Bacillus licheniformis

\begin{abstract}
In this study, we characterized the heat shock stimulon of the important industrial microorganism Bacillus licheniformis using DNA microarrays. While sharing a high degree of homology with the closely related model organism Bacillus subtilis, the heat shock stimulon of $B$. licheniformis exhibited several novel and unexpected features. Most notably, heat shock in B. licheniformis resulted in decreased amounts of mRNA from the $y \operatorname{tr} A B C E F$ operon, encoding a putative acetoin uptake system, and stimulated the transcription of purine biosynthesis and iron uptake genes. Unexpectedly, deletion of the $y$ trEF genes did not affect acetoin uptake, but increased heat sensitivity. To investigate the connection between heat stress and iron uptake further, we analyzed the iron limitation response of $B$. licheniformis by DNA microarrays and concluded that the response mostly involves the genes related to iron uptake and metabolism, while the only heat shock gene affected by iron limitation was clpE. We also attempted
\end{abstract}

to delete the fur gene (encoding the ferric uptake repressor), but unexpectedly found it to be essential in B. licheniformis. Using the fluorescent protein-encoding reporter gene under control of the dhb promoter, which responded to both heat shock and iron-starvation, we confirmed the overlap between these responses.

Copyright $\odot 2010$ S. Karger AG, Basel

\section{Introduction}

The Gram-positive bacterium Bacillus licheniformis is widely used in the industry as a host for the production of commercially interesting enzymes [Wang et al., 2005; Mohapatra et al., 2007]. It is well known for high-level protein secretion and is generally regarded as safe. However, production of heterologous proteins in large quantities is often a stressful event which may lead to protein misfolding and degradation or even cell death [Gasser et al., 2008]. The optimization of bacteria for high-level production is therefore an ongoing process that focuses on all cellular aspects which influence protein quality and quantity such as transcription, translation, folding, secre-

\section{KARGER}

Fax +41 613061234 E-Mail karger@karger.ch www.karger.com
(C) 2010 S. Karger AG, Basel

1464-1801/10/0183-0162\$26.00/0

Accessible online at:

www.karger.com $/ \mathrm{mmb}$
Allan K. Nielsen

Novo Allé 1LS.26

DK-2880 Bagsværd (Denmark)

Tel. +45 4446 2544, Fax +45 44467303

E-Mail aknn@novozymes.com 
tion, and stability [Sahdev et al., 2008]. Bacteria have developed elaborate cellular quality control systems that efficiently cope with damaged proteins. These include molecular chaperones and ATP-dependent proteases, both induced under various conditions of stress, including heat shock [Helmann et al., 2001; Nonaka et al. 2006]. Stress-induced proteins can potentially be exploited as yield boosters in cell factories by either coproduction of putative chaperones with a protein of interest or by deletion of putative proteases which degrade the commercially interesting product. Detailed knowledge of the heat stress response of $B$. licheniformis is therefore of paramount importance for industrial strain development.

The primary structure of most heat shock proteins appears to be highly conserved during evolution, suggesting that they exert a similar function in all organisms [Gupta et al., 1993]. Induction of heat shock genes in the model Gram-positive organism Bacillus subtilis occurs primarily at the level of transcription [Helmann et al., 2001]. These genes are organized in several regulons (classes), which together constitute the heat shock stimulon [Hecker et al., 1996; Helmann et al., 2001]. The class I heat shock genes of $B$. subtilis consist of the heptacistronic dnaK and the bicistronic groE operons. Both operons are under the negative control of the heat-responsive transcriptional repressor protein HrcA. These operons encode the major chaperones of the cytosol, the DnaK and the GroE systems, which interact with unfolded proteins and mediate their refolding in an ATP-dependent manner [LaRossa et al., 1991]. The class II heat shock genes of B. subtilis code for general stress proteins. All of these genes are under the positive control of $\sigma^{\mathrm{B}}$ and they are not only induced by heat, but also by ethanol, salt, acid, oxidation, desiccation and by starvation for oxygen, glucose, and phosphate. This is the largest group of heat-responsive genes, and the precise role of most of them is not clear, but they are believed to serve a general and nonspecific protective function during stress and starvation [Petersohn et al., 2001; Price et al., 2001]. The class III heat shock genes of B. subtilis consist of the tetracistronic $\operatorname{clp} C$ operon and the two single genes $c_{p} P$ and $c l p E$. ClpC and ClpE function either as parts of proteolytic complexes with the ClpP protease or as molecular chaperones on their own. Expression of the $c l p$ genes is regulated by the negative regulator CtsR [Derré et al., 1999]. The gene $h t p G$ is regulated differently from other identified heat shock genes and is the only member of class IV identified so far. This gene is under the positive control of an unidentified transcriptional activator. Little is known about the role of HtpG (Hsp90) in bacteria, but its homologs in yeast, and more complex eukarya, have chaperone-like activities [Versteeg et al., 2003]. The class V heat shock genes of B. subtilis comprise the CssRS twocomponent system and the two putative membranebound proteases HtrA and HtrB (YvtA). This system also responds to secretion stress, and the two proteases are believed to serve the role of removing the misfolded or aggregated proteins from the membrane-cell wall interface [Darmon et al., 2002]. Class VI comprises a large group of heat-responsive genes whose regulation is unknown, but different from the above-mentioned systems. This clear distinction of different classes of heat shock genes should be taken with some reserve, for the regulatory systems governing their expression may be overlapping. A good example of such an overlap is the interaction between $\sigma^{\mathrm{B}}$ and HrcA in Listeria monocytogenes [Hu et al., 2007].

$B$. licheniformis shares most of the heat shock genes with $B$. subtilis, and in this study we set out to map the heat shock regulon of this important industrial organism using DNA microarrays. We were able to confirm the key regulatory roles of the heat shock repressors HrcA and CtsR also in B. licheniformis, but we also encountered some unexpected regulations. For example, the $y$ tr operon of $B$. licheniformis (homologous to an acetoin uptake system in B. subtilis) [Yoshida et al., 2000] was repressed under heat shock, but proved to be critical for survival under the same conditions. Moreover, genes involved in iron uptake and metabolism were surprisingly upregulated by heat shock. These genes belong to the ferric uptake repressor (Fur) regulon in B. subtilis [Ollinger et al., 2006], parts of which are either nonresponsive or repressed under general stress [Price et al., 2001]. This discrepancy incited us to characterize the Fur regulon in B. licheniformis, at first by deleting fur, encoding Fur. However, introducing this deletion proved to be impossible under all the experimental conditions tested, and we therefore conclude that Fur is essential in B. licheniformis. Instead, we investigated the iron starvation response using a combination of transcriptomics and bioinformatic analyses. Finally, we chose one of the operons responding to both iron limitation and heat shock, $d h b$, to demonstrate this double control using fluorescent protein fusions.

\section{Results and Discussion}

Defining the Heat Shock Regulon of B. licheniformis

In order to elucidate the global transcriptional response of $B$. licheniformis to heat shock, DNA microarrays composed of oligonucleotides representing the 4,082 identified ORFs of the sequenced genome of $B$. lichenifor- 
Table 1. Transcriptional induction of B. licheniformis ATCC 14580 genes measured 10 and 30 min after transfer from 37 to $50^{\circ} \mathrm{C}$ and percent identity of their gene product to the closest homolog in B. subtilis

\begin{tabular}{|c|c|c|c|c|c|c|c|}
\hline \multirow{2}{*}{$\begin{array}{l}\text { Gene ID in } \\
\text { B. licheniformis }\end{array}$} & \multirow{2}{*}{$\begin{array}{l}\text { B. subtilis } \\
\text { homolog }\end{array}$} & \multirow[t]{2}{*}{ Identity, \% } & \multicolumn{2}{|c|}{ Time after heat shock } & \multirow[t]{2}{*}{$h r c A^{1}$} & \multirow[t]{2}{*}{$c t s R^{1}$} & \multirow[t]{2}{*}{ Putative gene product and function } \\
\hline & & & $10 \mathrm{~min}$ & $30 \mathrm{~min}$ & & & \\
\hline \multicolumn{8}{|l|}{ HrcA regulon } \\
\hline BL2094 & $h r c A$ & 82 & 2.9 & & -14 & & Transcriptional regulator \\
\hline BL2096 & $\operatorname{grp} E$ & 73 & 3.0 & & 2.4 & & Molecular chaperone \\
\hline BL2097 & dnaK & 95 & 2.4 & & 2.6 & & Molecular chaperone \\
\hline BL2098 & $d n a J$ & 88 & 1.5 & & 1.6 & & Molecular chaperone \\
\hline BL3283 & groEL & 96 & 6.7 & 5.4 & 4.7 & & Molecular chaperone \\
\hline BL3284 & groES & 95 & 5.9 & 5.1 & 3.7 & & Molecular chaperone \\
\hline \multicolumn{8}{|l|}{ CtsR regulon } \\
\hline BL3258 & ctsR & 88 & 2.3 & & & -16.0 & Transcriptional regulator \\
\hline BL3259 & $m c s A$ & 73 & 3.7 & & & 5.5 & ctsR modulator \\
\hline BL3260 & $m c s B$ & 87 & 2.6 & & & 6.9 & ctsR modulator \\
\hline BL3261 & $\operatorname{clpC}$ & 96 & 5.4 & & & 4.5 & ATP-dependent protease \\
\hline BL3640 & $c l p E$ & 81 & 3.9 & & & 42.0 & ATP-dependent protease \\
\hline BL3605 & $c l p P$ & 92 & 3.0 & & & 3.1 & ATP-dependent protease \\
\hline BL3262 & sms & 91 & 1.7 & & & 3.5 & DNA repair \\
\hline \multicolumn{8}{|l|}{ Ytr operon } \\
\hline BL0008 & $y \operatorname{trA}$ & 86 & -8.1 & -8.3 & & -2.8 & Acetoin transport (putative) \\
\hline BL0009 & $y \operatorname{tr} B$ & 77 & -9.3 & -7.6 & & -2.6 & Acetoin transport (putative) \\
\hline BL0010 & $y \operatorname{trC}$ & 47 & -2.4 & -3.6 & & -1.9 & Acetoin transport (putative) \\
\hline BL0011 & $y \operatorname{trE}$ & 81 & -11.4 & -9.0 & & -2.9 & Acetoin transport (putative) \\
\hline BL0012 & $y \operatorname{trF}$ & 62 & -8.8 & -7.8 & & -3.0 & Acetoin transport (putative) \\
\hline \multicolumn{8}{|l|}{ Iron metabolism } \\
\hline BL4020 & $d h b A$ & 67 & & 2.2 & & & Siderophore synthesis \\
\hline BL4023 & $d h b B$ & 59 & & 4.0 & & & Siderophore synthesis \\
\hline BL4021 & $d h b C$ & 63 & & 2.3 & & & Siderophore synthesis \\
\hline BL4022 & $d h b E$ & 75 & & 2.4 & & & Siderophore synthesis \\
\hline BL4024 & $d h b F$ & 68 & & 2.5 & & & Siderophore synthesis \\
\hline BL4016 & feuA & 74 & & 3.9 & & & Siderophore transport \\
\hline BL4017 & $f e u B$ & 80 & & 2.2 & & & Siderophore transport \\
\hline BL4018 & feuC & 72 & & 2.0 & & & Siderophore transport \\
\hline BL0703 & fhuB & 71 & & 1.7 & & & Iron transport \\
\hline BL0704 & fhu $G$ & 63 & & 2.3 & & & Iron transport \\
\hline BL1760 & $y c l Q$ & 59 & & 3.5 & & & Iron transport (putative) \\
\hline BL3470 & $y f i Y$ & 67 & 5.1 & 7.2 & & & Iron transport (putative) \\
\hline BL3080 & $y f k M$ & 82 & 2.4 & & & & Unknown \\
\hline BL1086 & $y h f Q$ & 64 & 2.6 & 2.4 & & & Putative iron transport \\
\hline BL4019 & besA & 63 & & 2.0 & & & Iron release \\
\hline BL4025 & $y b d Z$ & 59 & 2.4 & & & & Unknown \\
\hline BL0187 & $y x e B$ & 75 & 2.3 & & & & Putative iron transport \\
\hline \multicolumn{8}{|c|}{ Purine metabolism } \\
\hline BL1478 & purB & 95 & & 8.1 & & & Purine biosynthesis \\
\hline BL1479 & purC & 87 & & 2.6 & & & Purine biosynthesis \\
\hline BL1487 & purD & 82 & & 3.7 & & & Purine biosynthesis \\
\hline BL1476 & purE & 88 & & 6.6 & & & Purine biosynthesis \\
\hline BL1483 & purF & 93 & & 8.8 & & & Purine biosynthesis \\
\hline BL1486 & purH & 91 & & 8.8 & & & Purine biosynthesis \\
\hline BL1477 & purK & 78 & & 4.1 & & & Purine biosynthesis \\
\hline BL1481 & purL & 90 & & 4.2 & & & Purine biosynthesis \\
\hline BL1484 & purM & 85 & & 11.2 & & & Purine biosynthesis \\
\hline BL1485 & purN & 76 & & 2.8 & & & Purine biosynthesis \\
\hline BL1482 & purQ & 91 & & 9.8 & & & Purine biosynthesis \\
\hline
\end{tabular}


Table 1 (continued)

\begin{tabular}{|c|c|c|c|c|c|c|}
\hline \multirow{2}{*}{$\begin{array}{l}\text { Gene ID in } \\
\text { B. licheniformis }\end{array}$} & \multirow{2}{*}{$\begin{array}{l}\text { B. subtilis } \\
\text { homolog }\end{array}$} & \multirow[t]{2}{*}{ Identity, \% } & \multicolumn{2}{|c|}{ Time after heat shock } & \multirow[t]{2}{*}{$h r c A^{1} \quad c t s R^{1}$} & \multirow[t]{2}{*}{ Putative gene product and function } \\
\hline & & & $10 \mathrm{~min}$ & $30 \mathrm{~min}$ & & \\
\hline \multicolumn{7}{|c|}{ Other stress genes } \\
\hline BL3742 & $h t r A$ & 64 & 4 & 9.2 & & Serine protease \\
\hline BL0734 & $h t r B$ & 64 & 3.1 & 2.4 & & Serine protease \\
\hline BL0247 & $h t p G$ & 84 & 4.1 & 2.4 & & Molecular chaperone \\
\hline BL0946 & $y w c J$ & 63 & & 4.5 & & Transporter \\
\hline BL2479 & alsD & 67 & & 5.1 & & $\alpha$-Acetolactate synthase \\
\hline BL2480 & alsS & 76 & & 3.3 & & $\alpha$-Acetolactate decarboxylase \\
\hline BL3162 & $\operatorname{csp} B$ & 98 & & -2.3 & & Major cold shock protein \\
\hline BL0924 & $c y d B$ & 74 & & -2 & & Cytochrome Bd \\
\hline BL0925 & $\operatorname{cydC}$ & 72 & & -2.3 & & $\mathrm{ABC}$ transporter \\
\hline BL0926 & $c y d D$ & 67 & & -2.4 & & $\mathrm{ABC}$ transporter \\
\hline BL1943 & katE & 78 & 2.4 & & & Catalase 2 \\
\hline BL1558 & ори $A B$ & 81 & & -2.1 & & Glycin-betaine $\mathrm{ABC}$ transporter \\
\hline BL1556 & opuAC & 72 & & -2.4 & & Glycin-betaine $\mathrm{ABC}$ transporter \\
\hline BL2819 & $y d a G$ & 74 & 3.2 & & & General stress protein \\
\hline
\end{tabular}

Induction folds are listed as averages obtained from 2 experiments for each comparative analysis. - indicates downregulation of genes following heat shock.

${ }^{1}$ Differential regulation of genes in $h r c A$ and $c t s R$ strains relative to wt at $37^{\circ} \mathrm{C}$.

mis [Rey et al., 2004] were employed. Exponentially growing cells of $B$. licheniformis were exposed to heat stress at $50^{\circ} \mathrm{C}$, and cells were harvested for RNA isolation after 10 and $30 \mathrm{~min}$. RNA from heat-shocked and control cultures (not heat shocked) were reverse transcribed in the presence of either Cy3-dCTP or Cy5-dCTP and hybridized to glass-based microarrays as described in Experimental Procedures. A list of genes that are significantly regulated by heat stress was generated from two biological replicate experiments, each with 16 technical replicate values for each gene. Only genes that were found to be significantly regulated at a $99 \%$ confidence level in both biological replicate experiments were included in the final list. A list of all genes with significant regulation is presented in table 1 , and in online supplementary table 1 (www.karger.com/doi/10.1159/000315457).

Our experimental setup was not optimal for detecting the $\sigma^{\mathrm{B}}$ regulon, since $\sigma^{\mathrm{B}}$ induction by heat shock is known to be transient and brief (3-5 min) [Helmann et al., 2001]. However, a number of upregulated genes likely to be under the control of $\sigma^{\mathrm{B}}$ did appear in our dataset (table 1). These are characterized by the promoter consensus (-35GTTTAA...-10GGGWAW), as described in Helmann et al. [2001] and Petersohn et al. [2001]. A detailed study of the $\sigma^{\mathrm{B}}$ regulon in $B$. licheniformis is underway by another group [Hecker M, personal commun.] and we did not pursue it further. In our experiment, we also identified upregulation of class IV genes (HtpG operon) and class V genes (CssRS regulon; table 1). Surprisingly, a large set of purine biosynthetic genes was also upregulated by heat shock. This observation might be related to one or more of these enzymes being heat-sensitive, thus necessitating the upregulation of the whole set of genes to maintain the proper rate of purine biosynthesis.

Finally, we focused in more detail on the HrcA and CtsR regulons, and two unexpected classes of genes regulated by heat shock: the upregulated iron metabolism genes and the downregulated putative acetoin uptake genes that seemed likely to constitute a species-specific response of $B$. licheniformis.

HrcA and CtsR Regulons of B. licheniformis

The class I heat shock genes of $B$. subtilis consist of the heptacistronic dnaK and the bicistronic groE operons, both under the negative control of the heat-responsive transcriptional repressor protein $\mathrm{HrcA}$. HrcA binds to conserved operator sequences known as CIRCE elements: in $B$. subtilis these comprise two perfect inverted repeats of 9 bp separated by a 9-bp spacer with the consensus sequence TTAGCACTC-N ${ }_{9}$-GAGTGCTAA [Zuber and 
Fig. 1. Characterization of the HrcA regulon. Transcriptome of the $\Delta h r c A$ strain (A) and the $\Delta h r c A$ strain complemented from the xylose-inducible promoter (B). Units on axes are $\log 2$ of relative expression levels.
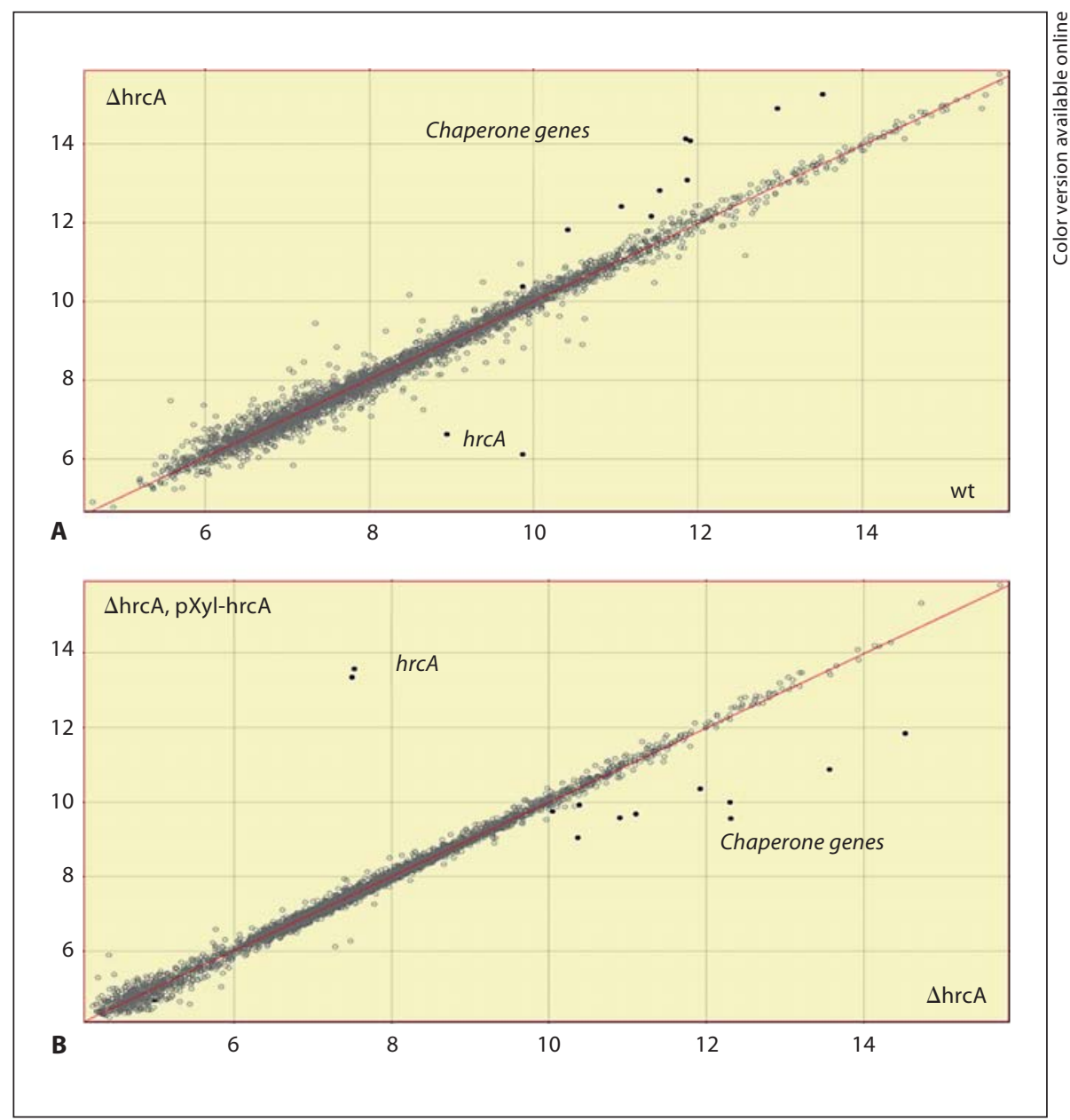

Schumann, 1994; Wiegert et al., 2003]. Our bioinformatic analyses of the B. licheniformis genome have identified two canonical CIRCE elements; one followed by a putative $d n a K$ operon and the other followed by a putative groE operon. The genes of the putative operons are organized in a subtilis-like manner, share homology with corresponding heat shock genes of $B$. subtilis, and have a transient nature of heat-responsive gene expression (table 1). To confirm the HrcA regulon, we inactivated the $h r c A$ gene and repeated the DNA array analysis, without heat shock but including a complementation strain with the $h r c A$ gene under the control of a xylose-inducible promoter. As demonstrated in figure $1 \mathrm{~A}$, genes encoding molecular chaperons are overexpressed in the $\Delta h r c A$ strain even without heat shock, and in the hrcA-complemented strain the regulation is reversed, the chaperonencoding genes are repressed, which probably reflects the fact that complementation strain contains elevated amounts of HrcA compared to the wild-type strain (fig. 1B).

CtsR is the negative regulator of the class III heat shock genes of $B$. subtilis which encode the clp proteins that form proteolytic complexes and may also function as molecular chaperones [Derré et al., 1999]. Our search of the $B$. licheniformis genome for the CtsR consensus binding site A/GGTCAAANANA/GGTCAAA revealed the expected protease-encoding genes. The inactivation of the $c t s R$ gene and its complementation from the xylose-inducible promoter allowed us to define the CtsR regulon (fig. 2, compare A to B). Interestingly, proteases were upregulated in the ctsR mutant as expected, but some other genes, such as the $y \operatorname{tr}$ operon putatively involved in acetoin uptake, were downregulated, implying that $y$ tr genes are somehow positively regulated by CtsR. 
Fig. 2. Characterization of the CtsR regulon. Transcriptome of the $\Delta c t s R$ strain (A) and the $\Delta c t s R$ strain complemented from the xylose-inducible promoter (B). Units on axes are $\log 2$ of relative expression levels.
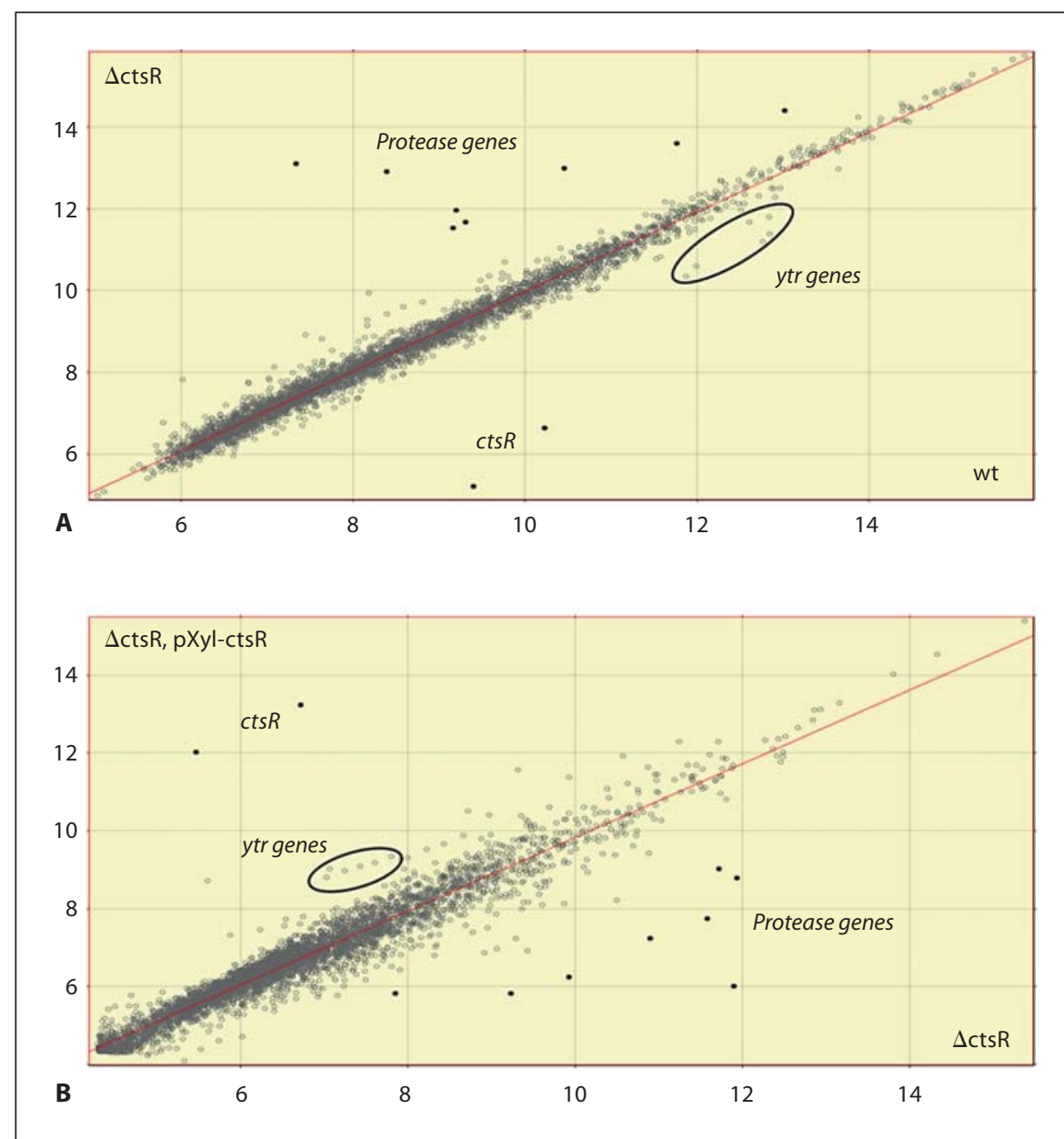

The B. licheniformis ytr Operon Is Required for Heat Resistance

In B. subtilis, the $y$ tr genes encode a putative system for the uptake of acetoin, an extracellular carbon storage compound that is produced during exponential growth and imported specifically at the onset of stationary growth phase [Yoshida et al., 2000]. This operon in $B$. subtilis was suggested to be under control of YtrA, a gene regulator from the GntR family [Rigali et al., 2002]. However, the inducer for the acetoin operon in B. subtilis remains unknown [Yoshida et al., 2000]. In B. lichenifor$m i s$, we found the $y$ tr genes to be downregulated by heat shock, and they were repressed in the $\Delta c t s R$ strain in the absence of heat shock. Since we found no CtsR binding site upstream of the $y$ tr operon, we suggest that the regulation proceeds in an indirect manner. CtsR could be repressing a repressor of $y$ tr genes, and this negative regulation could proceed via $c l p$ proteins (repressed by CtsR).
ClpP was found to modulate the activity of $\sigma^{\mathrm{B}}$ in B. subtilis [Reeves et al., 2007], and ClpE was found to modulate the CtsR repression in Lactococcus lactis [Varmanen et al., 2003], therefore it would not be unprecedented if $c l p$ proteins exerted an effect on gene regulation. We prepared a knockout of the $y$ trEF genes in B. licheniformis, and found, unexpectedly, that the resultant strain (AEB956) was severely impaired in growth at high temperatures (fig. 3). We therefore had to conclude that despite the fact that $y$ tr expression is downregulated during heat shock, the activity of these genes is still very important and might play a role in heat resistance. To investigate whether the $y \operatorname{tr}$ genes are indeed acetoin transporters in B. licheniformis, we quantified the acetoin uptake in the wild-type and $\Delta y \operatorname{trEF}$ strains. In B. subtilis, a $\Delta y t r$ strain was impaired in acetoin uptake [Yoshida et al., 2000], but we could not see any effect of the $y$ trEF deletion on acetoin uptake in B. licheniformis (results not shown), 


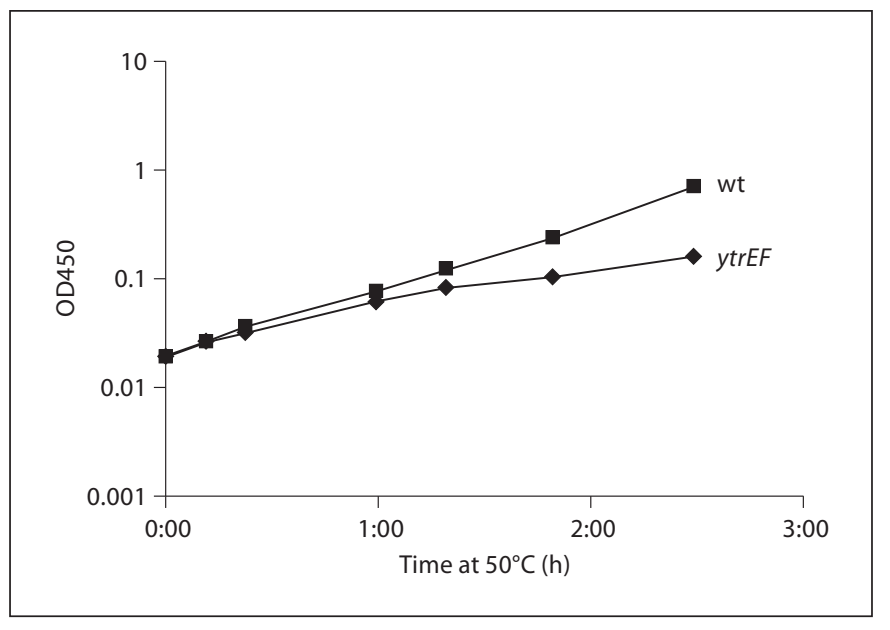

Fig. 3. Function of the $y t r$ operon. Growth of the $\Delta y t r E F$ strain $(\mathrm{AEB} 956, \boldsymbol{})$ compared to wild-type (ATCC14580, $\mathbf{a})$ during heat shock.

indicating that the function of this operon is different in this species.

\section{Defining the Iron Starvation Response of}

B. licheniformis

Bacterial iron homeostasis is regulated by the ferric uptake regulator Fur that acts mostly as a repressor of genes involved in iron uptake and respiratory chain enzymes [Ollinger et al., 2006]. The repression by Fur assures that the cell will stop taking up iron beyond a certain intracellular level, since excessive cytosolic iron would cause oxidative damage. Interestingly, five genes encoding iron-binding proteins ( $f e u A$, fhuD, yfiY, yfmC and $y \times e B$ ) were found to be repressed by general stress in B. subtilis [Price et al., 2001]. When our microarray data revealed quite the opposite, namely the induction of a number of genes involved in iron metabolism under the conditions of heat shock, we were prompted to investigate this further. B. licheniformis possesses a homolog of the Fur protein from B. subtilis. However, the B. licheniformis Fur seems to be more versatile in its function than its $B$. subtilis counterpart. Our attempts to knock out the $B$. licheniformis fur gene were futile, even when we attempted to do it in the iron-free medium. Only the presence of the ectopic fur gene under control of a xylose-inducible promoter made it possible to inactivate the chromosomal copy of fur. The essentiality of the fur gene indicates that its product has additional and important roles beyond those of its counterpart in B. subtilis.
As an alternative method to investigate the genes involved in iron metabolism in B. licheniformis, we performed DNA microarray analysis in the presence and absence of iron and found a number of genes to be upregulated during iron starvation, some of which are listed in table 2 , the remaining can be found in online supplementary table 2 . Genes were selected for table 2 based on two criteria: they were strongly upregulated during iron starvation (3.0-fold or higher), or they were more modestly upregulated but encode gene products that are expected to be involved in iron sensing or metabolism (based on homology). Furthermore, genes that are located in an operon with genes fulfilling the criteria above are included in table 2 as well.

In B. subtilis, expression of iron homeostasis genes is often controlled by binding of the Fur repressor dimer to a 15-bp (7-1-7) inverted repeat, named the 7-1-7 Fur-binding motif [Baichoo and Helmann, 2002]. To evaluate whether expression of the genes in table 2 could be regulated by Fur, we made a search of the $B$. licheniformis genome for the 7-1-7 Fur-binding motif. As can be seen from table 2, we found putative 7-1-7 Fur-binding motifs in front of a number of the genes that were affected by the level of iron in the microarray analysis, making it likely that expression of these genes is at least in part controlled by Fur.

Some of the genes most strongly upregulated during iron starvation belong to the dhbACEBF operon. This operon is homologous to the $d h b$ operon of $B$. subtilis which encodes proteins involved in the biosynthesis of the siderophore bacillibactin [Miethke et al., 2006]. Just upstream of $d h b A C E B F$ the $f e u A B C$ genes are located, which encode components of a ferri-bacillibactin uptake system, and which are also upregulated during iron starvation, though not as strongly as the $d h b$ operon. Since no region with the characteristics of a transcriptional terminator is found between the two groups of genes, it cannot be excluded that they belong to the same operon. Another strongly upregulated set of genes is the $y k u N O P$ operon, encoding flavodoxins; probably to provide an alternative to FeS-containing ferredoxins under iron-limiting conditions. Putative 7-1-7 Fur-binding motifs are located upstream of the $f e u A, d h b A$, and $y k u N$ genes.

A number of similarities exist between the Fur regulons in B. subtilis and B. licheniformis. One of the striking dissimilarities is an operon composed of the genes BL01336 to BL01331 in B. licheniformis. A putative Furbinding motif with only one mismatch was found upstream of the operon, and expression of the genes was induced up to fourfold during iron starvation (not shown). No obvious homologs of these genes were found in B. sub- 
Table 2. Transcriptional induction of B. licheniformis ATCC14580 genes during iron starvation and the percent identity of their gene product to the closest homolog in B. subtilis 168

\begin{tabular}{|c|c|c|c|c|c|}
\hline $\begin{array}{l}\text { Gene ID in } \\
\text { B. licheniformis }\end{array}$ & $\begin{array}{l}\text { B. subtilis } \\
\text { homolog }\end{array}$ & $\begin{array}{l}\text { Identity } \\
\%\end{array}$ & $\begin{array}{l}\text { Fold } \\
\text { induction }\end{array}$ & $\begin{array}{l}\text { Mismatches in 7-1-7 } \\
\text { Fur-binding motif }\end{array}$ & $\begin{array}{l}\text { Putative gene or operon product and function } \\
\text { in B. subtilis (if any) }\end{array}$ \\
\hline \multicolumn{5}{|c|}{ feuABC-dhbACEBF operon ${ }^{2}$} & Uptake and synthesis of bacillibactin \\
\hline BL04016 & feuA & 74 & 2.7 & 1 & Iron hydroxomate-binding protein \\
\hline BL04017 & $f e u B$ & 80 & 3.2 & - & Iron-uptake protein \\
\hline BL04018 & $\mathrm{feuC}$ & 73 & 2.0 & - & Iron-uptake protein \\
\hline BL04019 & besA & 63 & 2.6 & - & Bacillibactin trilacetone hydrolase \\
\hline BL04020 & $d h b A$ & 67 & 5.2 & 1,1 & Dihydroxybenzoate dehydrogenase \\
\hline BL04021 & $d h b C$ & 63 & 5.1 & - & Isochorismate synthase \\
\hline BL04022 & $d h b E$ & 75 & 9.9 & - & Enterobactin synthetase component E \\
\hline BL04023 & $d h b B$ & 60 & 8.5 & - & Isochorismatase \\
\hline BL04024 & $d h b F$ & 68 & 8.3 & - & Bacillibactin synthetase \\
\hline BL04025 & $y b d Z$ & 59 & 11.0 & - & \\
\hline BL04026 & $y h c A^{3}$ & 26 & 4.5 & - & Putative exporter \\
\hline \multicolumn{5}{|l|}{$y k u N O P$ operon } & Flavodoxin \\
\hline BL03588 & $y k u N$ & 65 & 4.8 & 0 & Short-chain flavodoxin \\
\hline BL03589 & $y k u O$ & 69 & 7.2 & - & \\
\hline BL03590 & $y k u P$ & 74 & 5.3 & - & Short-chain flavodoxin \\
\hline \multicolumn{5}{|l|}{ tenA-thiVWX operon } & Thiamine uptake and metabolism \\
\hline BL02647 & $\operatorname{ten} A$ & 35 & 2.7 & - & Thiaminase \\
\hline BL02648 & thiV & 35 & 3.0 & - & Thiamine transporter \\
\hline BL02650 & thiW & 36 & 2.3 & - & Thiamine $\mathrm{ABC}$ transporter (ATP-binding protein) \\
\hline BL02651 & thiX & 37 & 2.0 & - & Thiamine transporter, permease \\
\hline \multicolumn{5}{|l|}{ pdhA-D operon } & Pyruvate dehydrogenase \\
\hline BL01616 & pdhA & 82 & 2.9 & - & Pyruvate dehydrogenase \\
\hline BL01617 & $p d h B$ & 90 & 3.1 & - & Pyruvate dehydrogenase \\
\hline BL01618 & $p d h C$ & 79 & 1.6 & - & Pyruvate dehydrogenase \\
\hline BL01619 & $p d h D$ & 90 & 1.8 & - & Dihydrolipoamide dehydrogenase E3 subunit \\
\hline \multicolumn{6}{|l|}{$y w c-s a c T$ operon } \\
\hline BL05359 & $y w c$ & 31 & 3.0 & - & \\
\hline BL03889 & $\operatorname{sact}$ & 69 & 1.5 & - & Transcriptional antiterminator \\
\hline \multicolumn{5}{|l|}{$y c l N O P Q$ operon } & Putative ferrichrome $\mathrm{ABC}$ transporter \\
\hline BL01757 & $y c l N$ & 75 & 1.6 & 1,2 & Iron-siderophore transporter (permease) \\
\hline BL01758 & $y c l O$ & 70 & 2.9 & - & Iron-siderophore transporter (permease) \\
\hline BL01759 & $y c l P$ & 75 & 1.6 & - & Iron-siderophore transporter (ATP-binding protein) \\
\hline BL01760 & $y c l Q$ & 59 & 2.6 & & Iron-siderophore transporter (binding lipoprotein) \\
\hline \multicolumn{6}{|r|}{ 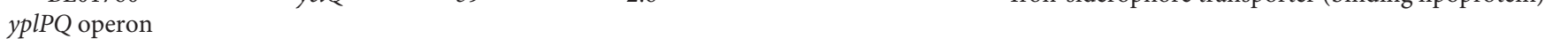 } \\
\hline BL03309 & yplP & 52 & 1.9 & $-{ }^{4}$ & Transcriptional enhancer \\
\hline BL03308 & yplQ & 80 & 3.8 & - & Putative membrane hydrolase \\
\hline \multicolumn{6}{|l|}{$Y k o L-Y d b N$ operon } \\
\hline BL05040 & $y k o L$ & 36 & 6.0 & 0,2 & \\
\hline BL02212 & $y d b N$ & 57 & 2.1 & - & \\
\hline \multicolumn{6}{|l|}{ Single genes } \\
\hline BL02042 & $y c g T$ & 40 & 7.9 & 1 & Putative thioredoxin reductase \\
\hline BL03470 & yfiY & 55 & 4.8 & 1 & Putative iron (III) dicitrate transporter lipoprotein \\
\hline BL03906 $6^{5}$ & dhas & 39 & 3.0 & - & Putative aldehyde dehydrogenase \\
\hline BL01086 & $y h f Q$ & 64 & 3.1 & 0 & Putative iron (III) dicitrate-binding protein \\
\hline BL00187 & $y x e B$ & 73 & 2.0 & 2 & $\mathrm{ABC}$ transporter (ferrioxamine binding lipoprotein) \\
\hline BL04062 & $c l p E$ & 81 & 2.0 & - & ATP-dependent protease \\
\hline BL00468 & ухев & 36 & 1.9 & 0,2 & $\mathrm{ABC}$ transporter (ferrioxamine binding lipoprotein) \\
\hline BL01579 & fhuD & 78 & 1.6 & - & Ferrichrome $\mathrm{ABC}$ transporter \\
\hline BL02894 & hmp & 48 & 1.6 & - & Flavohemoglobin \\
\hline
\end{tabular}

Induction folds are listed as averages obtained from 2 experiments for each comparative analysis. ${ }^{1}$ Number of mismatches in putative Fur box upstream of the gene (see text). Two numbers indicate the presence of two putative Fur boxes; - indicates that no Fur box with less than three mismatches could be identified. ${ }^{2}$ Expression of btr, the first gene in the Feu-Dhp operon (located just upstream of $f e u A$ ) is not regulated by iron or heat, and the gene is not preceded by a putative Fur box. In B. subtilis, Btr acts as a positive regulator of $f e u A B C$ expression [Gaballa and Helmann, 2007] and is transcribed from its own promoter with its own Fur box, independent of the feu operon. ${ }^{3}$ In other Bacilli (e.g. B. thurigensis), proteins with high homology to the BL04026 protein are predicted to belong to the EmrB/QacA drug resistance transporter family, the first member of which was characterized in E. coli [Furukawa et al., 1993]. ${ }^{4}$ A putative Fur box with two mismatches are located in the adjacent $i l v A$ gene, $640 \mathrm{bp}$ upstream of the $y p l P$ start codon. ${ }^{5}$ BL03906 is located as the first gene in an operon consisting of seven genes. No Fur-binding motifs are found in the operon, expression of the last six genes is only affected mildly or not at all by iron starvation, and they do not display homology to genes relevant for iron starvation. 
tilis, but a wider homology search revealed that they are probably part of a pathway for synthesis of a siderophore that exists in bacilli other than B. subtilis. Bacillus pumilis contains an operon (BPUM1019-1024) encoding proteins with high homology to B. licheniformis BL0133101336. E. coli contains a similar operon, and the gene products of the last four genes have been termed IucA-D and shown to be involved in the synthesis of the siderophore aerobactin [de Lorenzo et al., 1986].

The iron starvation-induced Fur regulon in B. licheniformis comprised mainly proteins involved in iron metabolism and did not provide any clues as to the essentiality of the Fur regulator. We therefore might assume that this protein has other regulatory functions, induced by presently unidentified stimuli. In addition, iron-regulated genes did not appear to be affected in knockouts of major heat shock regulators such as HtrA or CtsR (table 1), indicating that the Fur regulator itself might be the one to respond to heat stress.

\section{Case Study of the dhb Operon Confirms Double}

Regulation by Heat Shock and Iron Level

In order to validate our conclusions as to the double regulation of the iron uptake genes by heat shock and iron availability (both possibly acting via the Fur regulator), we devised an experiment that would independently confirm the DNA microarray data. We chose to focus on the $d h b$ regulon, which was significantly upregulated by heat shock, and the most upregulated one by iron starvation, according to transcriptomic data. We constructed a strain (MKZY23) in which the reporter gene encoding the green fluorescent protein (GFP) is inserted as the last gene of the $f e u A B C-d h b A C E B F$ operon. The reporter gene was thus placed under the control of the $d h b$ promoter, allowing us to test our conclusions also at the translational level. This strain was exposed to either heat shock or iron starvation in parallel to the wild-type. Even though two different patterns of induction were observed, in each case significant activation of the $f e u A B C-d h b A C E B F$ operon, i.e. synthesis of the GFP, was observed. During iron starvation, the induction of GFP production in MKZY23 was constant, at a level twofold higher than the background level of increased fluorescence observed when ATCC14580 was grown under iron limitation. However, when the cells were transferred from 37 to $50^{\circ} \mathrm{C}$, a transient induction of 2- to 3 -fold was observed in fluorescence before returning to the background level within 60 min after induction; a pattern of induction which is often observed during heat shock. Even though the level of induction is not as high as observed with the microarray analysis, this finding confirmed that the feuABC-dhbACEBF operon independently presents expression patterns typical of both heat shock and iron starvation responses. Whether both of these patterns depend on the Fur regulator still remains to be determined.

\section{Conclusions and Perspectives}

In this work we have characterized the heat shock response of B. licheniformis on the transcriptional level. Our findings indicate that the major classes of heat shock genes present in B. subtilis are also functional in B. licheniformis. However, B. licheniformis exhibits some specific and novel regulatory patterns in coping with heat shock. Namely, it represses the $y$ tr genes, even though the same genes are required for full heat resistance. Simultaneously, it overproduces purine biosynthesis enzymes and iron uptake proteins. The iron uptake genes are presumably controlled by the regulator Fur. One possible explanation for our results is that Fur responds to both iron levels and heat shock as the effectors. Interestingly, expression of the bacillibactin synthesis genes DhbA, DhbB, DhbC, and DhbE is upregulated by high salinity in B. subtilis in a Fur-dependent manner, providing an example from a closely related species where Fur is able to respond to more than one stimulus. However, one cannot exclude the possibility that heat shock itself could induce iron starvation, as has been suggested in B. subtilis [Hoffmann et al., 2002]. While it is not essential in B. subtilis, Fur was found to be essential in $B$. licheniformis, as it is in Pseudomonas aeruginosa [Barton et al., 1996]. Fur in P. aeruginosa is however quite distinct from other Fur regulators, since it lacks the canonic zincbinding cysteine residues [Lewin et al., 2002] that are essential for protein folding, and contains a highly positively charged C-terminus. By contrast, multiple sequence alignment reveals a high degree of conservation between the non-essential Fur of B. subtilis and the essential Fur of B. licheniformis, making it impossible to make functional inferences from the primary structure. Further structural and functional analyses are needed to elucidate the functioning of the unusual Fur regulator from B. licheniformis.

\section{Experimental Procedures}

Strains and Growth Conditions

B. licheniformis strain ATCC14580 [Rey et al., 2004] and derivatives thereof were used in this work. When needed, $5 \mu \mathrm{g} / \mathrm{ml}$ erythromycin or $7 \mu \mathrm{g} / \mathrm{ml}$ tetracycline were added to the growth medium. Cells for heat shock induction experiments and other purposes were grown at $37^{\circ} \mathrm{C}$ in the TY medium. Exponentially 
growing cells of $B$. licheniformis were exposed to heat stress by transfer of culture flasks from 37 to $50^{\circ} \mathrm{C}$. The time taken to reach the final temperature was $5 \mathrm{~min}$ and cells were harvested for RNA isolation 10 and $30 \mathrm{~min}$ after the transfer. To measure GFP levels during heat shock, 200- $\mu$ l samples were withdrawn from the growth experiment both before and after transfer to $50^{\circ} \mathrm{C}$, and the GFP level was determined. Minimal medium [Antelmann et al., 1997] was used for iron starvation (with or without $1 \mu \mathrm{M} \mathrm{FeSO}$ ). For microarray analysis, B. licheniformis was grown in the presence and absence of iron, and the samples were harvested after 3-5 generations of exponential growth. For measurement of GFP levels during iron limitation, 200- $\mu$ l samples were withdrawn throughout the growth experiment and the GFP level was measured.

Genetic modifications of ATCC14580 included knockouts of heat shock regulators, knockout complementation strains and strains with the reporter gene encoding GFP, as listed in table 3. Gene knockouts were performed as follows: upstream and downstream regions of genes to be deleted were amplified by SOE-PCR and cloned in a mobilizable plasmid vector containing oriT and erm from pUB110 [Gryczan et al., 1978] and the temperature-sensitive replicon from $\mathrm{pE} 194$ [Iordanescu, 1976]. The constructs were transferred from $B$. subtilis to $B$. licheniformis by conjugation, resulting in a double crossing-over [Sahdev et al., 2008]. This procedure was used to construct the strains MOL2424 ( $h r c A$ ), JA1913 (ctsR), JA1917 ( $h r c A c t s R)$ and AEB956 (ytrEF). hrcA (MOL2424) and $c t s R$ (JA1913) knockout strains were complemented by expression of $h r c A$ (AN314) or ctsR (AN339), respectively, from a chromosomally located xylose-induced expression system. When the system is integrated in the chromosome it contains, in $5^{\prime}-3^{\prime}$ direction: the xylose repressor encoded by $x y l R$, the promoter and $5^{\prime}$ portion of $x y l A, x y l \mathrm{R} / \mathrm{A}$ intergenic operator sequences, a multiple cloning site containing the cloned gene of interest, eGFP and a $x y l B$ intragenic region. A gene of interest cloned in the multiple cloning site is therefore under control of the xylose-inducible $x y l A$ promoter and expressed from a dicistronic operon with the downstream gene encoding eGFP. Strain MKZY23 contains the gene encoding the GFP (eGFP) inserted as the last gene of the feuABCdhbABCEF-BL04025-BL04026 operon. The 753 bps downstream the $B L 04026$ (excluding the stop codon) and the 906 bps upstream the BL04026 stop codon (including the stop codon) were amplified by PCR. eGFP and the chloramphenicol resistance marker (cat) were co-amplified using a Novozymes A/S in-house plasmid as a template for the PCR. The three PCR products were joined by SOE-PCR and integrated in the chromosome by a double crossover event as described above, resulting in strain MKZY23.

\section{DNA Microarray Fabrication}

Microscope glass slides were cleaned for $2 \mathrm{~h}$ in a solution containing $9 \%(\mathrm{w} / \mathrm{v}) \mathrm{NaOH}$ and $35 \%(\mathrm{v} / \mathrm{v})$ ethanol. They were coated by soaking for $1 \mathrm{~h}$ in a poly-L-lysine solution containing $15 \%$ $(\mathrm{v} / \mathrm{v})$ poly-L-lysine and $0.1 \times \mathrm{PBS}$, washed 3 times in water, dried by centrifugation $\left(500 \mathrm{rpm}, 5 \mathrm{~min}, 20^{\circ} \mathrm{C}\right.$ ) and stored for at least 1 week before use. A total of 4,082 unmodified 48- to 52-oligomers, representing all identified ORFs of the $B$. licheniformis genome, were purchased from MWG Biotech AG (Ebersberg, Germany) and diluted in $50 \%(\mathrm{v} / \mathrm{v})$ DMSO to obtain concentrations of $50 \mathrm{pmol} / \mu \mathrm{l}$. B. licheniformis microarrays were printed on polyL-lysine-coated glass slides at Novozymes A/S (Bagsvaerd) using a Microgrid II arrayer (BioRobotics, Huntingdon, UK) followed by UV cross-linking at $60 \mathrm{~mJ}$ in a UVStratalinker 1800 (Biocrest
Table 3. List of B. licheniformis strains used in this work

\begin{tabular}{llll}
\hline Strain & Relevant genotype & Comments & $\begin{array}{l}\text { Source/ } \\
\text { reference }\end{array}$ \\
\hline ATCC14580 & B. lich wt & & $\begin{array}{l}\text { Rey et al. } \\
2004\end{array}$ \\
MOL2424 & $h r c A$ & $\Delta(\mathrm{nt} 7-745)$ & this work \\
JA1913 & $c t s R$ & $\Delta(\mathrm{nt}$ 15-464) & this work \\
JA1917 & $h r c A, c t s R$ & & this work \\
AN314 & $h r c A ;$ xyl::Pxyl-hrcA-gfp & this work \\
AN339 & $c t s R ;$ xyl::Pxyl-ctsR-gfp & this work \\
AEB956 & $y t r E F$ & this work \\
MKZY23 & $d h b A C E B F-B L 04026-$ & & this work \\
& $B L 04025-g f p-c a t$ & \\
\hline
\end{tabular}

BV, Amsterdam, The Netherlands). Free poly-L-lysine groups were neutralized by washing the slides for $15 \mathrm{~min}$ in 1-methyl-2pyrrolidinone containing $1.7 \%(\mathrm{w} / \mathrm{v})$ succinic anhydride and $4.3 \%(\mathrm{v} / \mathrm{v})$ boric acid ( $\mathrm{pH} 8.0)$ followed by washing 3 times in water. Slides were blocked by washing in $1 \%(\mathrm{w} / \mathrm{v})$ bovine serum albumin, $4 \times \mathrm{SSC}(3 \mathrm{M} \mathrm{NaCl}$ plus $3 \mathrm{M}$ sodium citrate) and $0.5 \%$ (w/v) SDS, for $45 \mathrm{~min}$ at $42^{\circ} \mathrm{C}$ followed by washing 5 times in water and drying by centrifugation $\left(500 \mathrm{rpm}, 5 \mathrm{~min}, 20^{\circ} \mathrm{C}\right)$. Each of the 4,082 ORFs was present 4 times on the $B$. licheniformis genome chip.

RNA Isolation, cDNA Labeling, and Hybridization

Total RNA was isolated from cell pellets using the High Pure RNA Isolation Kit (1828665; Roche). RNA was further purified using acid-phenol:chloroform, 5:1, pH 4.5 (9720, Ambion) and concentrated by precipitation with ethanol. RNA samples were quantified spectrophotometrically at $260 \mathrm{~nm}$ and quality was checked by the integrity of the $23 \mathrm{~S}$ and $16 \mathrm{~S}$ rRNA bands on an agarose gel. Conversion of $20 \mu \mathrm{g}$ RNA to cDNA and cDNA labeling was performed using the CyScribe Post-Labelling Kit (RPN5660X; Amersham Biosciences) according to the manufacturer's instructions. Sample cDNA (treated culture) was mixed with the corresponding control cDNA (untreated culture). Probes in $3 \times \mathrm{SSC}(0.45 \mathrm{M} \mathrm{NaCl}$ and $0.45 \mathrm{M}$ sodium citrate $)$ containing $0.3 \%$ sodium dodecyl sulfate (SDS) and $0.5 \mu \mathrm{g} / \mu \mathrm{l}$ tRNA were denatured at $90^{\circ} \mathrm{C}$ for $2 \mathrm{~min}$ and finally cooled on ice for a few seconds, immediately followed by application onto the chip. Labelled cDNA was hybridized to B. licheniformis chips under a supported cover slip in a humid chamber overnight at $60^{\circ} \mathrm{C}$ in a dark water bath. After hybridization, the cover slip was gently removed by placing the chip in a buffer of $2 \times$ SSC and $0.1 \%$ SDS. The chip was washed for $5 \mathrm{~min}$ in $2 \times$ SSC $+0.1 \%$ SDS, for $5 \mathrm{~min}$ in $1 \times$ SSC $+0.1 \%$ SDS, for $5 \mathrm{~min}$ in $0.5 \times$ SSC, for $10 \mathrm{~s}$ in deionized water and finally dried by centrifugation $(500 \mathrm{rpm}, 5 \mathrm{~min}$, $\left.20^{\circ} \mathrm{C}\right)$.

\section{Data Analysis}

Probed arrays were scanned at 532 and $635 \mathrm{~nm}$ at a $10-\mu \mathrm{m}$ resolution with a GMS 418 array scanner (Genetic Micro Systems) and a quantitative analysis of hybridization patterns and inten- 
sities was performed using Imagene 5 software (BioDiscovery, USA). Datasets were Lowess print-tip normalized and merged in GeneSight 3.5 (BioDiscovery, USA). Finally, the data were validated using the Confidence Analyzer Tool (99\% confidence level) in GeneSight 3.5. Four technical replicate hybridizations $(2 \times$ dye shift) were made for each time point. Since each probe is present 4 times on the DNA chips, this generates two biological replicate datasets, each containing 16 technical replicate values for each gene. Only genes that were found to be significantly regulated at the $99 \%$ confidence level in both biological replicate experiments were included in the final list.

\section{Measurements of Fluorescent Protein (GFP)}

$200 \mu \mathrm{l}$ of each sample for fluorescence measurement was taken directly from the growing culture and immediately chilled on icing water. $100 \mu \mathrm{l}$ of each sample was applied to a black microti- ter plate (Nunc) and measured using an automated fluorescence microplate reader (Fluorostar Galaxy, BMG Lab Technologies, Germany) fitted with 485-nm excitation and 520-nm emission filters.

\section{Quantification of Acetoin}

$1-\mathrm{ml}$ aliquots of cells were removed at regular intervals during the growth experiment. Cells were removed by centrifugation and the acetoin concentration in the supernatant was determined as described previously [Grundy et al., 1993].

\section{Acknowledgement}

We thank Annette Hansen for expert technical assistance.

\section{References}

Antelmann H, Engelmann S, Schmid R, Sorokin A, Lapidus A, Hecker M: Expression of a stress- and starvation-induced dps/pexBhomologous gene is controlled by the alternative sigma factor sigmaB in Bacillus subtilis. J Bacteriol 1997;179:7251-7256.

Baichoo N, Helmann JD: Recognition of DNA by Fur: a reinterpretation of the Fur box consensus sequence. J Bacteriol 2002;184:58265832 .

Barton HA, Johnson Z, Cox CD, Vasil AI, Vasil ML: Ferric uptake regulator mutants of $P$ seudomonas aeruginosa with distinct alterations in the iron-dependent repression of exotoxin $\mathrm{A}$ and siderophores in aerobic and microaerobic environments. Mol Microbiol 1996;21:1001-1017.

-Darmon E, Noone D, Masson A, Bron S, Kuipers OP, Devine KM, van Dijl JM: A novel class of heat and secretion stress-responsive genes is controlled by the autoregulated CssRS twocomponent system of Bacillus subtilis. J Bacteriol 2002;184:5661-5671.

de Lorenzo V, Bindereif A, Paw BH, Neilands JB: Aerobactin biosynthesis and transport genes of plasmid ColV-K30 in Escherichia coli K-12. J Bacteriol 1986;165:570-578.

-Derré I, Rapoport G, Msadek T: CtsR, a novel regulator of stress and heat shock response, controls clp and molecular chaperone gene expression in gram-positive bacteria. Mol Microbiol 1999;31:117-131.

Furukawa H, Tsay JT, Jackowski S, Takamura Y, Rock CO: Thiolactomycin resistance in Escherichia coli is associated with the multidrug resistance efflux pump encoded by emrAB. J Bacteriol 1993;175:3723-3729.

-Gaballa A, Helmann JD: Substrate induction of siderophore transport in Bacillus subtilis mediated by a novel one-component regulator. Mol Microbiol 2007;66:164-173.
Gasser B, Saloheimo M, Rinas U, Dragosits M, Rodríguez-Carmona E, Baumann K, Giuliani M, Parrilli E, Branduardi P, Lang C, Porro D, Ferrer P, Tutino ML, Mattanovich D, Villaverde A: Protein folding and conformational stress in microbial cells producing recombinant proteins: a host comparative overview. Microb Cell Fact 2008;7:11.

- Grundy FJ, Waters DA, Takova TY, Henkin TM: Identification of genes involved in utilization of acetate and acetoin in Bacillus subtilis. Mol Microbiol 1993;10:259-271.

Gryczan TJ, Contente S, Dubnau D: Characterization of Staphylococcus aureus plasmids introduced by transformation into Bacillus subtilis. J Bacteriol 1978:134:318-329.

-Gupta RS, Golding GB: Evolution of HSP70 gene and its implications regarding relationships between archaebacteria, eubacteria, and eukaryotes. J Mol Evol 1993;37:573-582.

Hecker M, Schumann W, Völker, U: Heat-shock and general stress response in Bacillus subtilis. Mol Microbiol 1996;19:417-428.

Helmann JD, Wu MF, Kobel PA, Gamo FJ, Wilson M, Morshedi MM, Navre M, Paddon C: Global transcriptional response of Bacillus subtilis to heat shock. J Bacteriol 2001;183: 7318-7328.

Hoffmann T, Schütz A, Brosius M, Völker A, Völker U, Bremer E: High salinity-induced iron starvation in Bacillus subtilis. J Bacteriol 2002; 174:718-727.

Hu Y, Oliver HF, Raengpradub S, Palmer ME, Orsi RH, Wiedmann M, Boor KJ: Transcriptomic and phenotypic analyses suggest a network between the transcriptional regulators $\mathrm{HrcA}$ and sigma B in Listeria monocytogenes. Appl Environ Microbiol 2007;73:7981-7991.

Iordanescu S: Three distinct plasmids originating in the same Staphylococcus aureus strain. Arch Ruom Pathol Exp Microbiol 1976;35: 111-118.
LaRossa RA, Van Dyk TK: Physiological roles of the DnaK and GroE stress proteins: catalysts of protein folding or macromolecular sponges? Mol Microbiol 1991;5:529-534.

Lewin AC, Doughty PA, Flegg L, Moore GR, Spiro S: The ferric uptake regulator of Pseudomonas aeruginosa has no essential cysteine residues and does not contain a structural zinc ion. Microbiology 2002;148:2449-2456.

-Miethke M, Klotz O, Linne U, May JJ, Beckering CL, Marahiel MA: Ferri-bacillibactin uptake and hydrolysis in Bacillus subtilis. Mol Microbiol 2006;61:1413-1427.

Mohapatra PK, Mondal KC, Pati BR: Production of tannase by the immobilized cells of Bacillus licheniformis KBR6 in Ca-alginate beads. J Appl Microbiol 2007;102:1462-1467.

Moszer I, Glaser P, Danchin A: SubtiList: a relational database for the Bacillus subtilis genome. Microbiology 1995;141:261-268.

Nonaka G, Blankschien M, Herman C, Gross CA, Rhodius VA: Regulon and promoter analysis of the E. coli heat-shock factor, sigma32, reveals a multifaceted cellular response to heat stress. Genes Dev 2006;20: 1776-1789.

- Ollinger J, Song KB, Antelmann H, Hecker M, Helmann JD: Role of the Fur regulon in iron transport in Bacillus subtilis. J Bacteriol 2006;188:3664-3673.

- Petersohn A, Brigulla M, Haas S, Hoheisel JD, Völker U, Hecker M: Global analysis of the general stress response of Bacillus subtilis. J Bacteriol 2001;183:5617-5631.

Price CW, Fawcett P, Cérémonie H, Su N, Murphy CK, Youngman P: Genome-wide analysis of the general stress response in Bacillus subtilis. Mol Microbiol 2001:41:757-774.

- Reeves A, Gerth U, Völker U, Haldenwang WG: ClpP modulates the activity of the Bacillus subtilis stress response transcription factor, sigmaB. J Bacteriol 2007;189:6168-6175. 
Rey MW, Ramaiya P, Nelson BA, Brody-Karpin SD, Zaretsky EJ, Tang M, Lopez de Leon A, Xiang H, Gusti V, Clausen IG, Olsen PB, Rasmussen $\mathrm{MD}$, Andersen JT, Jørgensen PL, Larsen TS, Sorokin A, Bolotin A, Lapidus A, Galleron N, Ehrlich SD, Berka RM: Complete genome sequence of the industrial bacterium Bacillus licheniformis and comparisons with closely related Bacillus species. Genome Biol 2004;5:R77.

Rigali S, Derouaux A, Giannotta F, Dusart J: Subdivision of the helix-turn-helix GntR family of bacterial regulators in the FadR, HutC, MocR, and YtrA subfamilies. J Biol Chem 2002;277:12507-12515.
Sahdev S, Khattar SK, Saini KS: Production of active eukaryotic proteins through bacterial expression systems: a review of the existing biotechnology strategies. Mol Cell Biochem 2008;307:249-264.

- Varmanen P, Vogensen FK, Hammer K, Palva A, Ingmer $\mathrm{H}$ : ClpE from Lactococcus lactis promotes repression of CtsR-dependent gene expression. J Bacteriol 2003;185:5117-5124.

-Versteeg S, Escher A, Wende A, Wiegert T, Schumann W: Regulation of the Bacillus subtilis heat shock gene htpG is under positive control. J Bacteriol 2003;185:466-474.
Wang JJ, Greenhut WB, Shih JC: Development of an asporogenic Bacillus licheniformis for the production of keratinase. J Appl Microbiol 2005;98:761-767.

Wiegert T, Schumann W: Analysis of a DNAbinding motif of the Bacillus subtilis HrcA repressor protein. FEMS Microbiol Lett 2003;223:101-106.

Yoshida KI, Fujita Y, Ehrlich SD: An operon for a putative ATP-binding cassette transport system involved in acetoin utilization of Bacillus subtilis. J Bacteriol 2000;182:5454-5461.

Zuber U, Schumann W: CIRCE, a novel heat shock element involved in regulation of heat shock operon dnaK of Bacillus subtilis. J Bacteriol 1994;176:1359-1363. 